\title{
INTERPRETATION OF DEFORMABLE MIRROR DEMONSTRATING MISSION (DeMi) CUBESAT: A REVIEW
}

\author{
Priyanka Khandagale \\ B.Tech, Department of Aerospace engineering \\ Sandip University Nashik India \\ Satya Sandeep Chaganti \\ Assistant Professor, Department of Aerospace engineering \\ Sandip University Nashik India
}

\begin{abstract}
The respective paper describes the design and implementation in a multi-actuator microelectromechanical system (MEMS) based deformable mirror (DM) for the $6 \mathrm{U}$ CubeSat Deformable Mirror Demonstration Mission (DeMi). The Deformable Mirror Demonstration Mission (DeMi) payload will specifically imply about 140 actuator MEMS.

Mainly two operational modes of DEMI payload are there, one mode that sees an internal illumination source and another manner that applies an external aperture to modify picture resolution and scale down the effects of atmospheric turbulence. DeMi is a project to demonstrate and characterize the performance of a deformable mirror in LEO on a $6 \mathrm{U}$ Cubesat. Space telescopes constructed with chronographs and wavefront control systems are very important to get the high contrast of $10^{10}$ to directly image an Earth-like exoplanet around a sun-like star at optical wavelengths. The contribution of this paper is, we have studied various important parts of the Deformable Mirror Demonstrating Mission, Deformable Mirrors, and its importance. In this report, we give an overview of Adaptive optics, Optical Design, Payload, operation, power, and sensors with the help of diagrams and board.
\end{abstract}

Keywords: Deformable Mirror Demonstrating Mission, Adaptive optics, cubist, Deformable Mirrors, Technology readiness levels, wavefront sensing, micro-electromechanical system (MEMS), Complementary Metal-oxide Semiconductor (CMOS) Camera.

\section{INTRODUCTION}

The CubeSat standard is an open specification for a household of small spacecraft formats. The California Polytechnic Institute (CalPoly) and Stanford University developed the Cube Sat form factor in 1999. It links with a common secondary payload deployed, the Poly-Pico satellite Orbital Deplorer (PPOD), which reduces the cost and time for obtaining launch opportunities for nanosatellites. The initial specification was for an approximately $10 \mathrm{~cm} \times 10$ $\mathrm{cm} \times 10 \mathrm{~cm}$ cube, now known as a single CubeSat unit (1U). Its existence has led to standardized spacecraft deployers which can be manifested on launch vehicles. Spacecraft can now more easily obtain a ride to orbit at a cut-price, while the launch provider has an avenue to monetize excess capacity on their vehicles which might otherwise only be filled with ballast. 10

\section{ADAPTIVE OPTICS}

During 1981, ADAPTIVE optics systems were brought in by the defense community and in 1991, extended to the scientific community after declassification that utilizes deformable mirrors.

The fundamental problem of Adaptive Optics (AO) is to correct the material body of a non-uniform incoming optical phase front. AO is typically employed for soil-based astronomy, where light from a distant object gets to the crest of the Earth's atmosphere effectively as a plane wave. The drawback of using a separate wavefront sensor is that it is situated separately from the primary optical path of the science instrument and then the illumination must be split using additional optical components. Misalignments or imperfections of elements in the 


\section{International Journal of Engineering Applied Sciences and Technology, 2020 Vol. 5, Issue 2, ISSN No. 2455-2143, Pages 241-250 \\ Published Online June 2020 in IJEAST (http://www.ijeast.com)}

optical paths induce additional phase aberrations in each wavefront, which is referred to as non-commonpath errors One manner to avoid these faults is by getting rid of the separate wavefront sensor and using the main optical sensor to assess the condition of the wavefront. AO is still important for a broad range of space telescope systems, even though they do not own to combat atmospheric distortion, because of manufacturing limitations and because of the challenging launch and distance environments.

On a smaller scale, AO is used in optometry to detect distortions in the lens of the human eye and to improve the imaging properties of optical microscopes in biological samples. In this study, we focus on the role of MEMS DMs with AO techniques for exoplanet direct imaging. ${ }^{10}$

\section{DeMi BACKGROUND}

Deformable mirrors correct imperfections, thermal distortions, and diffraction in the telescope and optics. DMs have a high actuator counts up to 128 across the pupil. Their high actuator density enables higher actuator counts for given pupil size. The reduction of pupil size allows a reduction of the size and weight of optics across the system, which is beneficial, which is a very essential technology for high-contrast imaging using future large space observatories. Microelectromechanical systems (MEMS) DMs are fitted to the task due to their high actuator density. MEMS DMs are appropriate for use in space because of their lower size, weight, and power (SWaP) than piezoelectric, electrostrictive, or voice coil designs also. Their low actuator mass makes them particularly resilient to launch-induced oscillations.

DMs can be used for many in-space applications, which include optical communication and wide-field scanning telescopes. It is also applicable for deployable, self-assembling, and other types of reconfigurable optical systems.

During a sub-orbital sounding rocket flight, a highactuator count MEMS DM has been operated widely, but additional recognition is required to prove that MEMS DMs are accurate for long-term in-space use of an operational telescope. The main requirement of performance for the DeMi payload is, it should calculate the surface of DM with an accuracy of $5 \mathrm{~nm}$ while wavefront sensing accuracy should be up to 10 nm.

The DeMi payload contains a DM driver and a Shack-Hartmann Wavefront Sensor (SHWFS) to evaluate the optical surface by wavefront reconstruction. An external aperture viewing a star, or an internal laser diode are two options for DM illumination. For the demonstration purpose, the omnibus contains a Cadet U UHF radio capable of receiving uplink and downlinking the data to an $18 \mathrm{~m}$ dish at the NASA Wallops Flight Facility at a rate of 1 Mbps ${ }^{1}$.

\section{Applications for Wavefront control system in space is as follows:}

(i) Submission of images through the Earth's turbulent atmosphere by the organizations,

(ii) Systems that carry and receives laser signals through the Earth's turbulent atmosphere,

Organizations that require high contrast as well as high dynamic range images of other targets in space.

(iv) Systems that transmit and receive laser signals from other objects in place.

In addition to their use, deformable mirrors can also be utilized as the amplitude or phase modulator that regulates the transmitted signals in free space laser communication systems. ${ }^{4,5}$

A variety of habits are found in microelectromechanical deformable mirror technology. From adaptive optics for correction of atmospheric turbulence and in-vivo imaging of the human retina to the design for a full-field scanning telescope and maximizing the contrast of a nulling interferometer for excellent imagery.

A single DM can correct phase and amplitude errors across half of a coronagraphic image. Promise beyond exoplanet imaging is held by deformable mirrors in space \& it includes many other applications such as laser communications, designable optical assemblies, or apertures that are deployable. The DeMi payload will characterize the functioning of a MEMS deformable mirror in low earth orbit, decreasing the technological risk to future applications. $^{2}$

DeMi is labor about demonstrating \& characterizing the execution of a deformable mirror in LEO on a $6 \mathrm{U}$ Cubesat. The heap, letting in the battery, the main information processing system, solar arrays, the communications systems, the spacecraft structure, and the attitude determination and control system (ADCS), are made up and traded as a commercial off-the-shelf (COTS) product by Blue Canyon Technologies of Boulder, Colorado ${ }^{3}$. 
The demi mission mainly focuses on producing a low cost, and easy access to space platform for checking the accuracy of technologies, the more complex, higher actuator count DMs5.

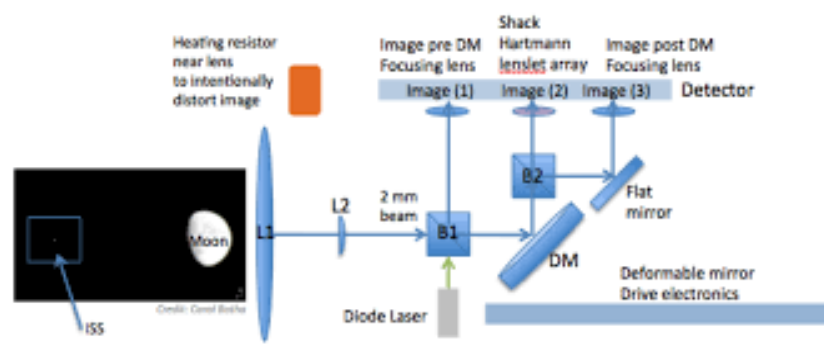

Fig 1: Demonstrating the function of wavefront control with a MEMS DM on a CubeSat. ${ }^{5}$

DeMi's mission is used to raise the Technology Readiness Level (TRL) of MEMS DMs from 5 to at least 7 . To judge the maturity of technologies during the learning phase of a program, the Technology readiness levels (TRLs) method is used. It is developed at NASA in the 1970s. The use of TRLs allows consistent, uniform discussions of technical maturity across different types of engineering. ${ }^{11}$ The key payload requirements are to calculate individual DM actuator wavefront displacement contributions to an accuracy of $12 \mathrm{NM}$, the low order optical aberrations which are up to $\lambda / 10$ accuracy and $\lambda / 50$ precision, and exact static and dynamic wavefront errors to less than $100 \mathrm{~mm}$ root-mean-square (RMS) error. The demo mission will show that MEMS DMs can survive the vibration, thermal, and radiation effects of launch and long duration operations in place. $^{6}$

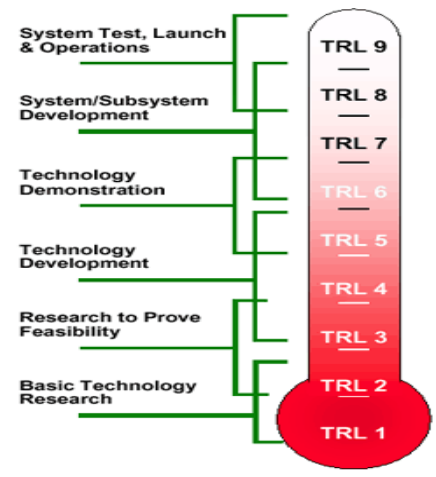

Fig 2: NASA Technology readiness levels ${ }^{11}$
IV.
RECOMMENDATIONS TO REDUCE RISKS

The evolution of a space telescope constructed with a high-performance chronograph as well as a deformable-mirror wavefront control system having $10^{10}$ contrasts needed to directly image. Earth-like exoplanets are assumed to be on the order of several hundreds of millions of dollars to over a billion dollars.

The CubeSat Deformable Mirror Demonstration mission aims to supply a low-cost path to quickly test small, low-power, higher actuator-count deformable mirror technologies on-orbit. Some essential environmental tests like a thermal vacuum, vibration, life cycle testing, radiation can be done by using these mirrors on the ground. It is very important to show that the simple wavefront control system using various new deformable mirror technologies have stable, well-calibrated, and predictable performance on orbit. This is essential to underline, as there is no chance to "tweak" or "adjust" a wavefront control system on-orbit after a launch. Fully develop robust flight software to control these mirrors and systems is of special importance; to integrate them as sensors with spacecraft attitude determination and restraint systems (ADCS), estimators, and fine pointing algorithms; and to pick up how best to capture performance and calibration data along with science observations and communicate it to the earth. ${ }^{4}$

\section{DESIGN}

\section{(i) Optical layout:}

The internal observation mode of DeMi uses an internal illumination source to examine the use of the adaptive optics systems. This is pictured in Figure 1. A small laser diode incorporated into the payload sends light through this character until it hits the field mirror ${ }^{3}$. There is an external aperture so a wizard can be seen, and there is an internal laser source (the red arrow is fiber launch) so that the mirror can be characterized even in the case that the space vehicle is unable to point at a champion. During external observation mode, light from a distant star enters through an aperture in the side of the spacecraft and is reflected off of an off-axis parabolic mirror (MI in Figure 3) towards the field mirror which is placed at the focal point of mirror M1. Light reflects off the field mirror to another mirror (M2 in Figure 3) which collimates the light and sends it towards the deformable mirror (DM in Figure 3). The light that is reflected off the deformable mirror travels through a beam-splitter, with half of the incoming light being reflected an image sensor through a lens (L1 in Figure 3) and the other half passing through a 
beamsplitter and then reflecting off of two more mirrors which form a pupil relay and then onto a Shack-Hartmann wavefront sensor 3,7.

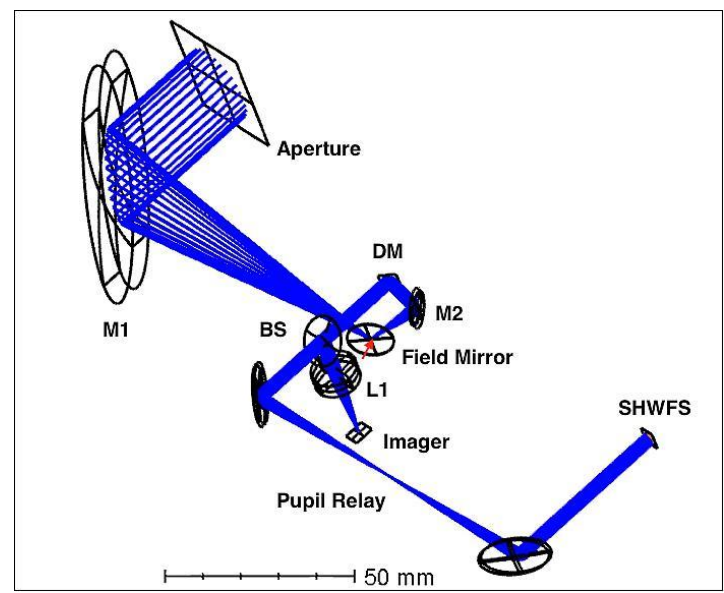

Fig 3: DeMi payload optical layout. ${ }^{1,3,7}$

(ii) Mechanical design:

The structural design of DeMi is specifically concerned about the versatility of the exact position of the optical element to keep it aligned. Due to the strength of the assembly to mechanical vibration, the repeatability of this alignment is also important and also the structure must have capable to align the optical elements, to survive a $100 \mathrm{~g}$ of load in all three axes, and maintain alignment through as much of the expected from $-5^{\circ} \mathrm{C}$ to $20^{\circ} \mathrm{C}$ thermal range as possible. $^{1}$

\section{(iii) Electrical and electronics design :}

The electronics stack, which is composed of the redundant payload computers, the deformable mirror driver boards, and the power supply board which generates the power rails need by the electronics stack.

To attach the DM driver boards with the DM, ribbon cables will be used. Power will be provided by the XB6 bus in three channels that can be independently switched by bus commands; one at $5 \mathrm{~V}$ and two at $3.3 \mathrm{~V}$. High-voltage about $250 \mathrm{~V}$ and $-5 \mathrm{~V}$ power for the DM controller will be generated on the power distribution board. To drive the internal laser diode, a constant-current power supply will be mounted beside the stack. A block diagram of the DeMi payload electronics is shown in Figure 4. ${ }^{1,6}$

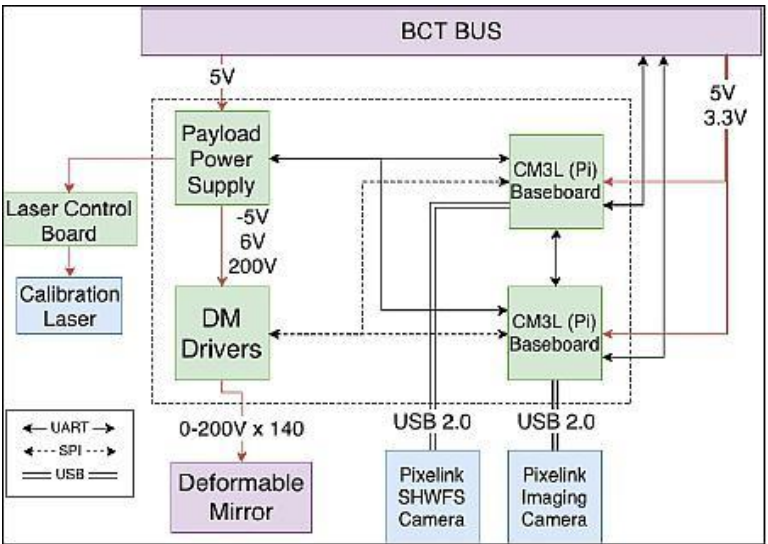

Fig 4. Block diagram of electronics system of DeMi. ${ }^{1}$

\section{POWER}

The bus controls power generation and storage and supplies $3.3 \mathrm{~V}$ and $5 \mathrm{~V}$ rails to the payload. Additional power processing for the calibration laser and the DM is controlled by the laser driver and DM driver, respectively. To actuate the DM, each channel must be supplied a variable voltage that can be as high as $250 \mathrm{~V}$. A hardware driver must be used to generate these voltages. ${ }^{2}$

Figures 5 and 6 show that, while peak power generation is higher with the deployed panels, the body-mounted panels provide a more uniform power generation pattern across the orbit. The use of only deployed panels does not provide a significant increase in power generation, but it makes for a less consistent power profile and deployed panels add unnecessary complexity and cost to the spacecraft. Hence, the current design employs four $3 \mathrm{U}$ bodymounted panels ${ }^{4}$.

\section{V.I. Deformable Mirror (DM):}

The CubeSat Deformable Mirror Demonstration aims to identify \& characterize the performance of a small deformable mirror over a year in low-Earth orbit. For low-power space-based laser communications, they can also improve distortions and reduce bit error rates.12 The DM in an $\mathrm{AO}$ system plays a role of correcting the wavefront for any aberrations or imperfections detected by the wavefront sensor and DM accomplishes this goal by deforming its shape into a conjugate of the detected wavefront. There are two main kinds of DMs, one is segmented, and the other is continuous. Segmented mirrors have individual flat surface mirrors attached to each actuator where continuous DM uses a continuous face-sheet mirror over the actuators. For its high 


\section{International Journal of Engineering Applied Sciences and Technology, 2020 \\ Vol. 5, Issue 2, ISSN No. 2455-2143, Pages 241-250 \\ Published Online June 2020 in IJEAST (http://www.ijeast.com)}

actuator count, large stroke, and good correctional capabilities, the 140 actuator multi DM was chosen within a reasonable cost.7

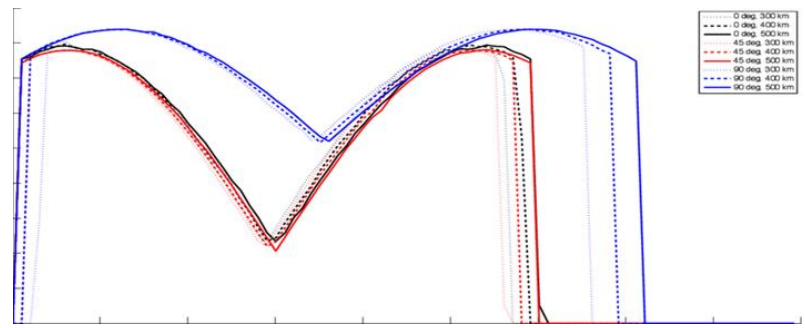

Fig 5: Power generated by $3 \mathrm{U}$ body (mounted solar panels throughout an orbit). 4

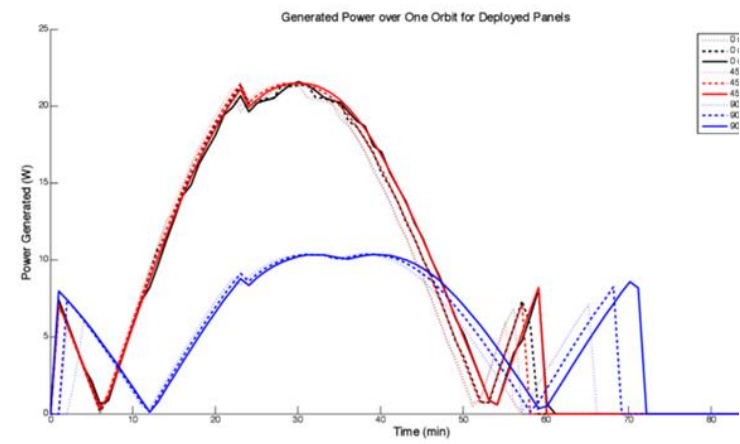

Fig 6: Power generated by four two-sided $3 \mathrm{U}$ deployable solar panels throughout an orbit4

\section{V.II Complementary Metal-Oxide Semiconductor (CMOS) Camera:}

CMOS camera is a technology used to produce integrated circuits. CMOS circuits are severally found in electronic components, including microprocessors, batteries, and digital camera image sensors.13 Two CMOS cameras are used on the DeMi payload, and both play major roles. One camera is used to capture the image from the observation and to direct the image plane wavefront sensing. The other camera is used in the SHWFS.7

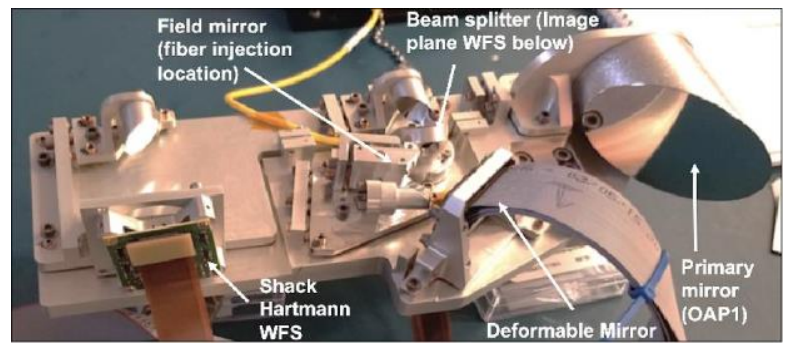

Fig 7: Aligned DeMi Engineering Model optical bench. Figure reproduced from Morgan et al. $2019^{6}$

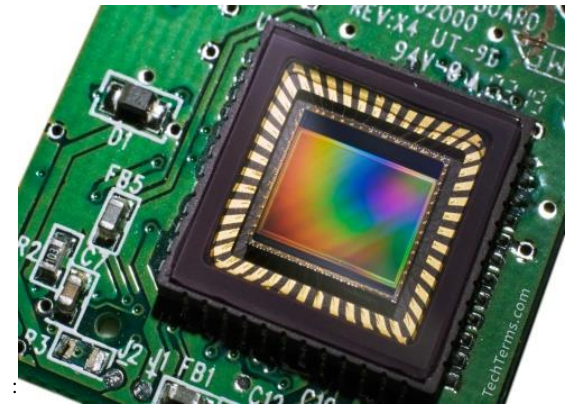

Fig 8: Coms camera ${ }^{14}$

\section{Payload :}

Federal Acquisition Regulation (FAR) defines the set of rules for COTS .9 The goal for the initial demonstration mission, is to use as much existing commercial off the shelf (COTS) hardware as possible, making improvements as needed or important for operation in space, and to keep the design as simple as possible. The simple purpose of the payload is the demonstration of the deformable mirror can be commanded and controlled on-orbit, to characterize its performance, and to show that its onorbit behavior is understandable as well as predictable. This aim can be accomplished by an internal coherent light source such as a laser diode, a deformable mirror, and a small number of static optical elements including a beamsplitter, a couple of collimating and focusing lenses, and a detector ${ }^{4,5}$.

\section{I Payload design trades}

The DeMi payload is an electro-optomechanical system hosted on the CubeSat bus. It contains the DM, optics to provide a stable wavefront, sensors to measure DM performance, and electronics to operate the DM, read out sensor data, and perform wavefront control. $^{10}$

To determine the exact configuration and most effective spacing of the optical elements within the payload, further modeling, and component-level trade studies are important. Assembly of a test-bench system in a laboratory setting will provide insight into the usefulness of the proposed design. One of the main key trades is the accurate selection of a MEMS $\mathrm{DM}$ and the corresponding mirror aperture. Although the deformable mirrors themselves are very small, even within their packaging, a vastly acknowledged challenge to incorporating high actuator count deformable mirror systems on a spacecraft is the substantial size, mass, volume, power, and complexity of the mirror driver boards and also wire 
harnesses. A "Mini" deformable mirror from the Boston Micromachines Corporation (BMC) is the current DeMi payload design. The most recently available BMC Mini packaging format is used for the DeMi mission. ${ }^{4}$

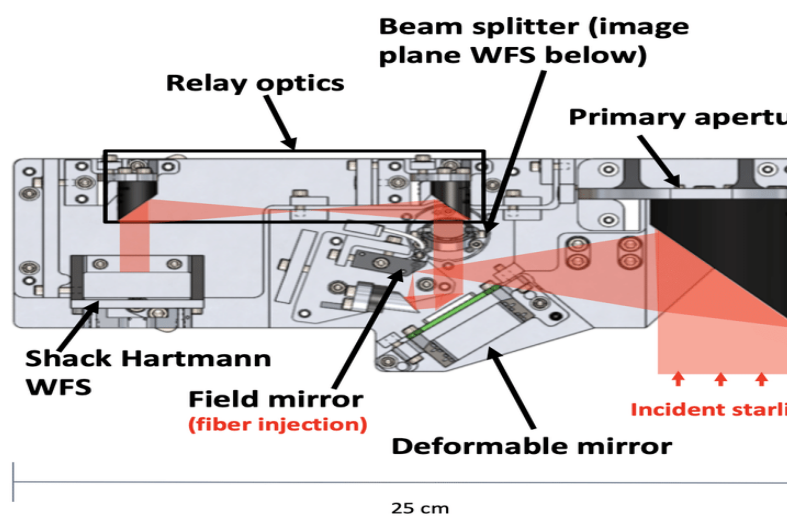

Fig 9: DeMi optical design with ray-trace overlaid in red. Figure reproduced from Morgan et al. $2019^{6}$

Uplink of spacecraft and payload commands and downlink of telemetry and science data will be accomplished through the use of two different RF communication links. An uplink frequency is about 449.75 MHz while downlink at 401.3 MHz. Cadet-U UHF radio will use high-rate data downlink, manufactured by the Space Dynamics Laboratory, and it will use a ground station at the NASA Wallops Flight Facility. With an uplink at $449.75 \mathrm{MHz}$ and downlink at $468 \mathrm{MHz}$, this link will operate at 3 Mbps. To downlink images and wavefront sensor data from the payload, as well as large blocks of payload and bus telemetry, the high-rate capability will be used. ${ }^{6}$

VI.II Payload design requirement :

\begin{tabular}{|c|c|c|c|c|}
\hline ID & Requirement & Description & Justification & Verification \\
\hline 1 & Channel Count & $\begin{array}{c}\text { The driver shall be able } \\
\text { to drive all channels } \\
\text { present on the Mirrorcle } \\
\text { Multi DM }\end{array}$ & $\begin{array}{c}\text { The success of } \\
\text { technology } \\
\text { demonstration payload }\end{array}$ & Analysis \\
\hline 2 & Dynamic range & $\begin{array}{l}\text { The driver shall have } \\
\text { sufficient dynamic } \\
\text { range to drive every } \\
\text { channel of the DM } \\
\text { from its resting state to } \\
\text { the desired stroke }\end{array}$ & $\begin{array}{c}\text { Success technology } \\
\text { demonstration payload } \\
\text { Success technology } \\
\text { demonstration payload }\end{array}$ & Analysis \\
\hline 3 & Resolution & $\begin{array}{l}\text { The driver shall produce } \\
\text { an output voltage on } \\
\text { each channel sufficient } \\
\text { to meet the actuator } \\
\text { mechanical }\end{array}$ & $\begin{array}{c}\text { Success technology } \\
\text { demonstration payload }\end{array}$ & Analysis \\
\hline 4 & Noise & $\begin{array}{l}\text { The driver shatt not } \\
\text { produce electrical noise } \\
\text { on its output that impact } \\
\text { its ability to the driver } \\
\text { every channel of the } \\
\text { DM to the desired } \\
\text { resolution }\end{array}$ & $\begin{array}{l}\text { Assist in the } \\
\text { characterization of DM } \\
\text { deflection curve } \\
\text { Compatibility with the }\end{array}$ & Analysis \\
\hline
\end{tabular}


International Journal of Engineering Applied Sciences and Technology, 2020

Vol. 5, Issue 2, ISSN No. 2455-2143, Pages 241-250

Published Online June 2020 in IJEAST (http://www.ijeast.com)

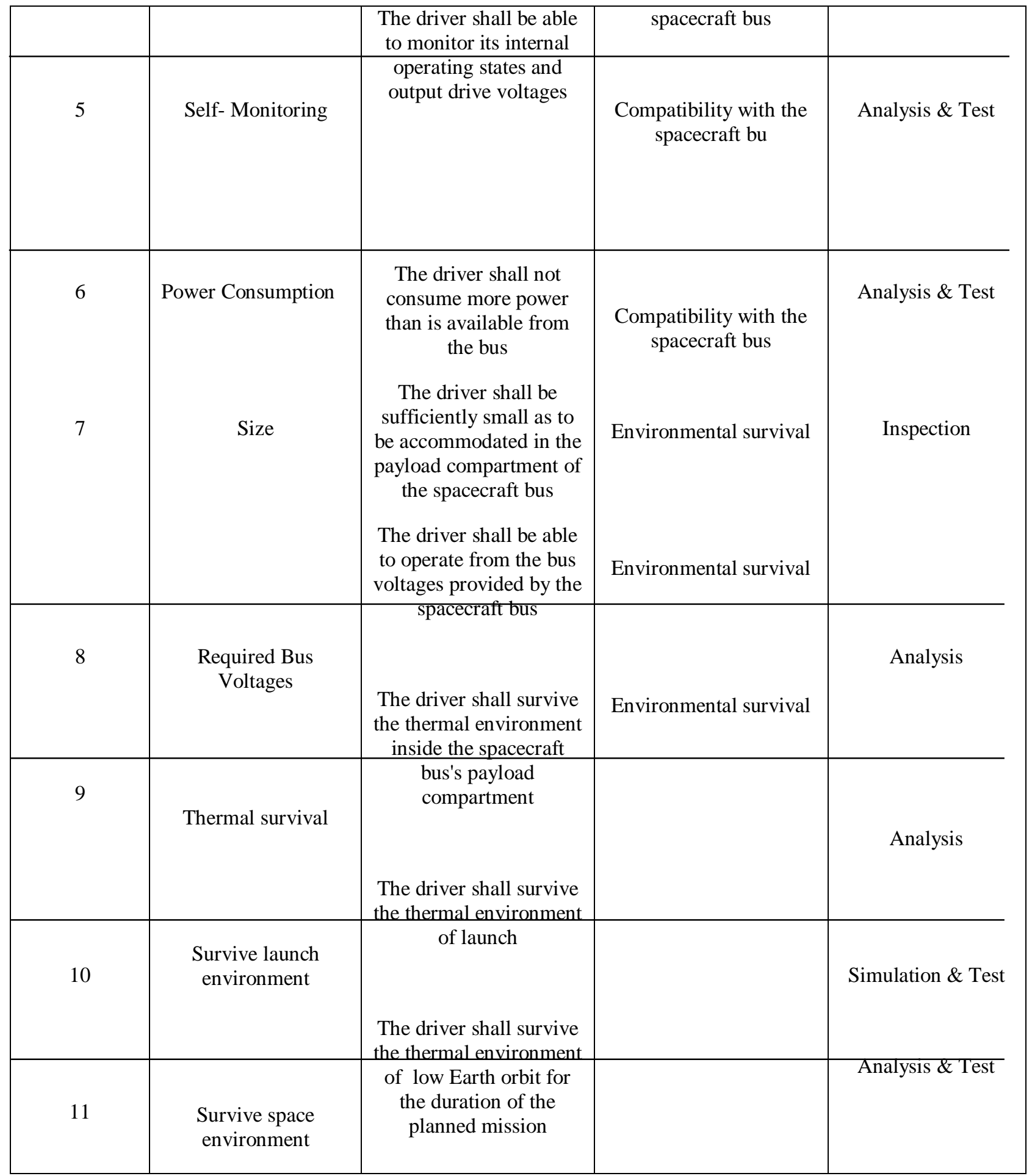

Table 1: DeMi Payload Driver Electronics Design Requirements ${ }^{3}$

VIII. OPERATIONS 
As a university nanosatellite project, it is desirable to maintain low complexity in design and operations. In addition to stars like Arcturus (HIP 69673) and Vega (HIP 91262), DeMi will image one of the brightest objects in the sky, the International Space Station (ISS). ${ }^{5}$ As spacecraft have different performance requirements to ensure that the spacecraft can safely and correctly perform its desired functions, before each operation, the spacecraft will perform several different checks for internal and external operations,. After all the checks, the spacecraft will power on the required components for the specific mode of operation. It will then test voltage and current to the components and finish by taking baseline measurements and image frames. ${ }^{5,7,10}$

\section{VII.I Internal Modes of Operation:}

Several system checks need to be done to ensure the successful operation of DeMi, for the internal operation. The spacecraft needs to ensure that the internal temperature, attitude control, data storage capacity, and power supply to the payload fit all the requirements. When the system checks pass, then the payload can power on the necessary components. For internal observations (fig 10), the following four components essential to being powered on Laser, DM, CMOS Camera \& SHWFS.

\section{VII.II External Mode of Operation:}

The spacecraft needs to perform more system checks, for external observations. In addition to the system checks needed for internal observations too, also spacecraft pointing and stability, as well as spacecraft position relative to the eclipse, need to check by the spacecraft. Once system checks are over, the spacecraft will need to power on the DM, CMOS camera, and the SHWFS. While testing the wavefront correction loop, the external mode of operation will perform astronomical observations. To look at the light source from stars and use the closed-loop wavefront correction system to demonstrate the correctional capabilities the external aperture will be used by this model. Figure 11 describes the steps required to complete external observation successfully.7

\section{SENSOR DESIGN}

The DeMi payload contains a custom DM driver and a Shack-Hartmann Wavefront Sensor (SHWFS) to measure the optical surface through wavefront reconstruction. The DeMi SHWFS is made up of a PixeLink PL-D775MU-BL CMOS camera board, a Thorlabs MLA150-5C-M microlens array, and a custom aluminum case that serve as a mount and a holder for the lenslet assembly10. The top view of DeMi SHWFS is as shown in Figure 12.

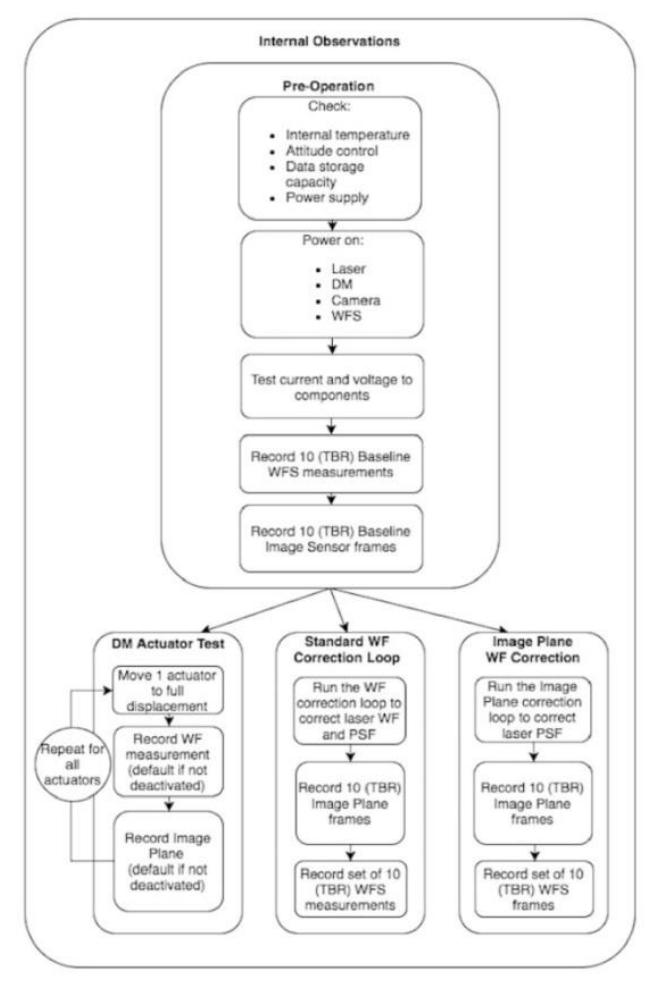

Fig 10: Internal modes of operation ${ }^{7}$ 
International Journal of Engineering Applied Sciences and Technology, 2020

Vol. 5, Issue 2, ISSN No. 2455-2143, Pages 241-250

Published Online June 2020 in IJEAST (http://www.ijeast.com)

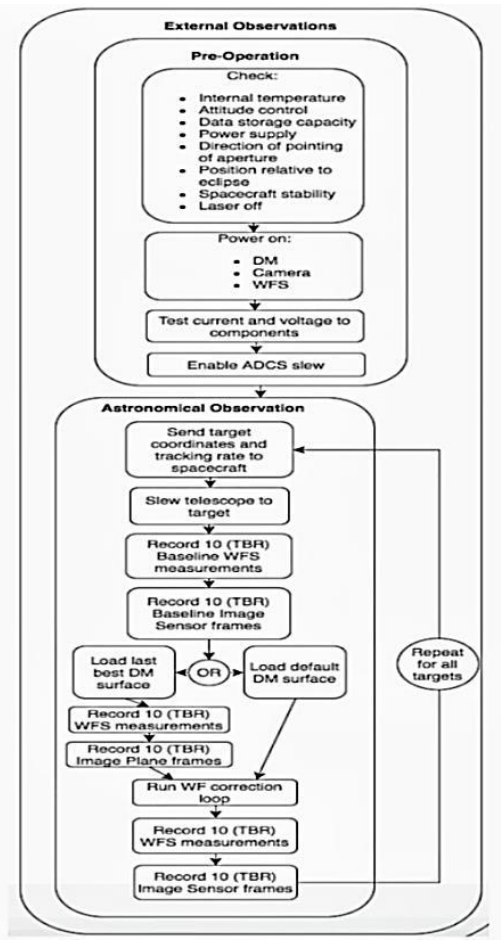

Fig 11: External operational mode ${ }^{7}$

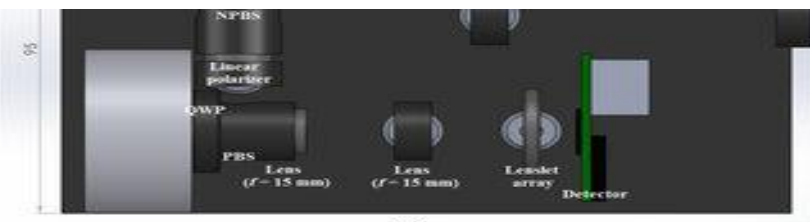

(a)

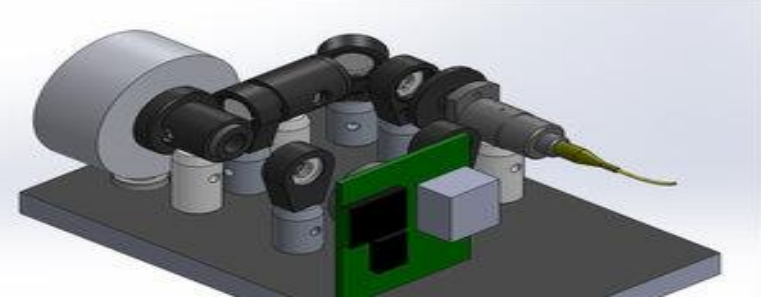

Fig 12: Top view of SHWFS

Shack-Hartmann wavefront sensors use a lenslet array to divide up the incoming beam of light and focus the divided beams on to a CMOS camera. The direction and shape of the incoming beam can be determined based on the displacement of the centroids from each beam in the divided array. An example of the SHWFS results for a distorted wavefront is as shown in Figure 137.
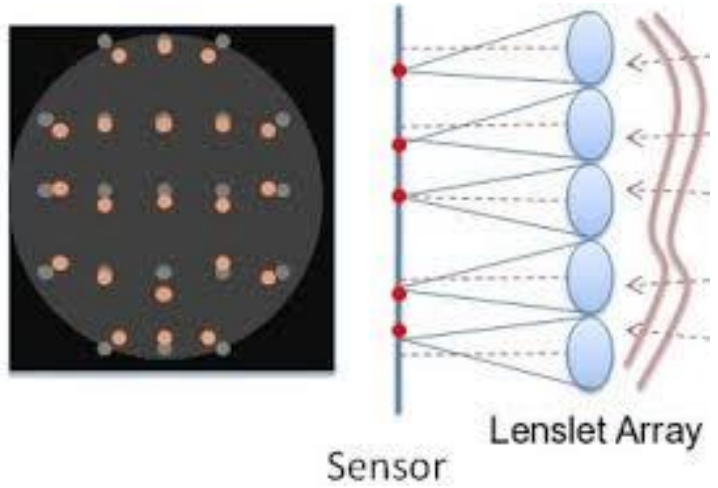

Fig 13: SHWFS simulation ${ }^{15}$

\section{CONCLUSION}

Respective paper is an overview of the Deformable Mirror Demonstration Mission (DeMi). This has provided a snapshot of the current design on the DeMi CubeSat payload. DeMi contains a simple resistive heating element to intentionally introduce small thermal distortions in the aperture so that their effects can be captured by the wavefront sensor and wavefront corrections applied using the MEMS deformable mirror. The detailed study of optical, mechanical, and electrical design, its operation with both internal and external modes, and also about payload design trades and requirements. Information about wavefront sensing, Deformable Mirror is explained in this paper. The main approach is to study each important component in detail and explaining it. The flight results from this mission will provide useful data for future space telescopes that plan to use MEMS deformable mirror technology.

\section{REFERENCES}

1. Allan, G., Douglas, E.S., Barnes, D., Egan, M., Furesz, G., Grunwald, W., Gubner, J., Haughwout, C., Holden, B.G., do Vale Pereira, P. and Stein, A.J., 2018, August. The deformable mirror demonstration mission (DeMi) CubeSat: optomechanical design validation and laboratory calibration. In Space Telescopes and Instrumentation 2018: Optical, Infrared, and Millimeter Wave (Vol. 10698, p. 1069857). International Society for Optics and Photonics.https://www.spiedigitallibrary.org /conference-proceedings-ofspie/10698/1069857/The-deformablemirror-demonstration-mission-DeMiCubeSat--optomechanicaldesign/10.1117/12.2314192.short 


\section{International Journal of Engineering Applied Sciences and Technology, 2020 \\ Vol. 5, Issue 2, ISSN No. 2455-2143, Pages 241-250 \\ Published Online June 2020 in IJEAST (http://www.ijeast.com)}

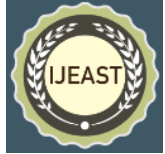

2. Douglas, E.S., Allan, G., Barnes, D., Figura, J.S., Haughwout, C.A., Gubner, J.N., Knoedler, A.A., LeClair, S., Murphy, T.J., Skouloudis, N. and Merck, J., 2017, September. Design of the deformable mirror demonstration CubeSat (DeMi). In Techniques and Instrumentation for Detection of Exoplanets VIII (Vol. 10400, p. 1040013). International Society for Optics and

Photonics.https://www.spiedigitallibrary.org /conference-proceedings-of-

spie/10400/1040013/Design-of-thedeformable-mirror-demonstration-CubeSatDeMi/10.1117/12.2274430.short

3. Haughwout, C.A., 2018. Electronics Development for the Deformable Mirror Demonstration Mission (DeMi) (Doctoral dissertation, Massachusetts Institute of Technology).https://dspace.mit.edu/handle/ 1721.1/120438

4. Cahoy, K., Marinan, A., Kerr, C., Cheng, K., and Jamil, S., 2012, September. CubeSat deformable mirror demonstration. In Space Telescopes and Instrumentation 2012: Optical, Infrared, and Millimeter Wave (Vol. 8442, p. 84424F). International Society for Optics and Photonics.https://www.spiedigitallibrary.org /conference-proceedings-ofspie/8442/84424F/CubeSat-deformablemirrordemonstration/10.1117/12.926681.short

5. Cahoy, K., Marinan, A., Kerr, C., Novak, B., Webber, M. and Kasdin, N.J., 2013, March. CubeSat Deformable Mirror Demonstration mission (DeMi). In 2013 IEEE Aerospace Conference (pp. 1-9). IEEE.https://ieeexplore.ieee.org/abstract/do cument/6497175/

6. Holden, B., Morgan, R., Allan, G., do Vale Pereira, P., Grunwald, W., Gubner, J., Haughwout, C., Stein, A., Xin, Y., Cahoy, K. and Douglas, E., 2019. Calibration and Testing of the Deformable Mirror Demonstration Mission (DeMi) CubeSat Payload.

https://digitalcommons.usu.edu/cgi/viewcon tent. cgi?article $=4370 \&$ context $=$ smallsat

7. Gubner, J., 2018. Payload Configuration, Integration, and Testing of the Deformable Mirror Demonstration Mission (DeMi) CubeSat.https://digitalcommons.usu.edu/sm allsat/2018/all2018/304/
8. Bandyopadhyay, S., Subramanian, G.P., Foust, R., Morgan, D., Chung, S.J., and Hadaegh, F., 2015. A review of impending small satellite formation flying missions. In 53rd AIAA Aerospace Sciences Meeting (p.

1623).https://arc.aiaa.org/DOI/pdf/10.2514/ 6.2015-1623

9. https://www.techopedia.com/definition/144 4/commercial-off-the-shelf-cots

10. Allan, G.W., 2018. Simulation and testing of wavefront reconstruction algorithms for the deformable mirror (DeMi) CubeSat (Doctoral dissertation, Massachusetts Institute of Technology).https://dspace.mit.edu/handle/ 1721.1/120381

11. Olechowski, A., Eppinger, S.D., and Joglekar, N., 2015, August. Technology readiness levels at 40: A study of state-ofthe-art use, challenges, and opportunities. In 2015 Portland international conference on management of engineering and technology (PICMET) (pp. 2084-2094). IEEE.https://ieeexplore.ieee.org/abstract/do cument/7273196/

12. https://www.nasa.gov/directorates/spacetech /strg/2012_nstrf_marinan.html\#: :text=The $\% 20$ goal $\% 20$ of $\% 20$ the $\% 20$ CubeSat,year $\% 2$ 0in\%20low\%2DEarth\%20orbit.\&text=The $\% 20$ payload $\% 20$ consists $\% 20$ of $\% 20$ a,wavef ront $\% 20$ sensor $\% 20$ and $\% 20 a \% 20$ detector.

13. https://www.google.com/url?sa=i\&url=https $\% 3 \mathrm{~A} \% 2 \mathrm{~F} \% 2$ Ftechterms.com\%2Fdefinition \%2Fcmos\&psig=AOvVaw1SYhpJQD1jRR ilITn9BR_w\&ust=1592546991564000\&sou rce $=$ images $\& c d=v f e \& v e d=0 C A 0 Q j h \times q F w o$ TCLCw7LLdiuoCFQAAAAAdAAAAABA D

14. https://techterms.com/img/lg/cmos_222.jpg

15. https://www.google.com/url?sa=i\&url=http $\% 3 \mathrm{~A} \% 2 \mathrm{~F} \% 2 \mathrm{Fiopscience.iop.org} \% 2 \mathrm{Farticle}$ $\% 2 \mathrm{~F} 10.1088 \% 2 \mathrm{~F} 17426596 \% 2 \mathrm{~F} 756 \% 2 \mathrm{~F} 1 \%$ 2F012002\%2Fpdf\&psig=AOvVaw3y3zKxu vpwYvgDiF9fWwyR\&ust=1592572126309 $000 \&$ source $=$ images $\& c d=v f e \& v e d=0 C A 0 Q$ jhxqFwoTCKCKpO66ioCFQAAAAAdAAAAABAD 